\title{
ON THE FACTORIZATION OF CREMONA PLANE
}

\section{TRANSFORMATIONS*}

\author{
BY \\ JAMES W. ALEXANDER
}

\section{INTRODUCTION}

1. Quite a number of results in algebraic geometry have been made to depend on Noether's fundamental theorem that a Cremona transformation of the plane can always be resolved into a product of quadratic ones. It is therefore desirable that this theorem be proved in as simple and convincing a manner as possible. Now, as was pointed out by Segre, $\dagger$ the proofs by Noether, Clifford, and others are incomplete and even break down for certain examples that can be effectively set up. There remains a very interesting proof by Castelnuovo $\ddagger$ which is especially designed to meet Segre's objections and which, along with a footnote to the same by Segre, suggested the method exposed in Part II below. The present proof, however, besides being considerably simpler and shorter, appears to be free from certain objections that might perhaps be raised to Castelnuovo's method. These objections, which are analogous to those directed against the older proofs, will be presented in Part I.

2. Let $N$ be the homaloidal net of a plane Cremona transformation and let $O_{0}, O_{1}, \cdots, O_{k}$ be the base points of $N$ arranged in order of decreasing multiplicities. Castelnuovo shows that a Jonquières transformation§ of suitable order $\nu$ having its base point of order $\nu-1$ at $O_{0}$ and its $2 \nu-2$ simple base points at $O_{1}, O_{2}, \cdots, O_{2 v-2}$ will, if it exists, reduce the net $N$ to one made up of curves of lower degree. Hence, if it can be shown that the transformation always exists, Noether's theorem will follow at once from the fact that every Jonquières transformation can be factored into quadratic ones. $\|$

* Presented to the Society, December 28, 1915.

† Segre, Atti della R. Accademia delle Scienzedi Torino, vol. 36 (1901), p. 645.

$\ddagger$ Castelnuovo, ibid., p. 861.

$\S$ Defined below.

\| See the proofs by Castelnuovo and Segre in the paper by the former already quoted. The proof in Part II does not make use of Jonquières transformations. 
Now, to prove the existence of the necessary Jonquières transformation, it must be shown (a) that there exist $\infty^{2}$ curves of order $\nu$ satisfying the given conditions at the base points, and $(b)$ that these curves are not all reducible. Under these conditions, the curves will make up the homaloidal net of the desired transformation. Castelnuovo appears to assume (a) tacitly in his proof of $(b),{ }^{*}$ making an assumption which might give rise to doubts when some of the base points $O_{i}$ are of the infinitely neighboring kind. $\dagger$ When the base points $O_{0}, O_{1}, \ldots, O_{2 \curvearrowleft-2}$ are all distinct, there can be no question whatsoever. The conditions imposed upon the curves of order $\nu$ cannot exceed $\nu(\nu-1) / 2+2 \nu-2=\left(\nu^{2}+3 \nu-4\right) / 2$, while there are $\infty^{\nu(\nu+3) / 2}$ curves of this order. Hence, there exist at least $\infty^{2}$ curves satisfying the conditions. But suppose the points $O_{2 \sim-2}$ and $O_{2 v-3}$ could both be infinitely neighboring to $O_{1}$ in the neighborhood of the first order. $\neq$ Then in order to pass through $O_{2 \downarrow-2}$ and $O_{2 \downarrow-3}$, a curve would necessarily have a double point at $O_{1}$, and the condition of passing through these three points would be expressed by 5 equations among the coefficients instead of by 3 as in the general case. We would then no longer expect to find $\infty^{2}$ curves satisfying all the conditions. A number of other cases are conceivable which, if they could actually arise, would lead to difficulties; we cite only two:

$(\alpha)$ Could not the sum of the multiplicities of those of the points $O_{1}, O_{2}$, $\cdots, O_{2 \nu-2}$ which were infinitely neighboring of the first order to $O_{0}$ be greater than $\nu-1$, imposing upon the curves of degree $\nu$ the condition of having at $O_{0}$ a multiple point of order greater than $\nu-1$ ?

$(\beta)$ When we impose on the curves of degree $\nu$ the condition of passing in the way assigned through $O_{0}, O_{1}, \cdots, O_{2 v-3}$ might they not all in consequence be tangent at $O_{1}$ to a fixed line $t$. If this were possible and if at the same time $O_{2 \sim 2}$ were infinitely neighboring to $O_{1}$ along a line other than $t$, the curves could not pass through $O_{2 \downarrow-2}$ without having a double point at $O_{1}$.

3. The objections that were raised in the last section will perhaps not seem over-critical if it is recalled that only the impossibility of making an assumption like (a) prevents one from reducing the order of a net by means of a single quadratic transformation. It is well known that the sum of the multiplicities of the three points $O_{0}, O_{1}$, and $O_{2}$ is greater than the order $n$ of the net $N$, so that if these points can be taken as the base points of a quadratic transformation, they will transform the net $N$ into one of lower order. Further-

\footnotetext{
*Castelnuovo, ". . ammesso che ciascuna delle $\infty^{2}$ curve $C \ldots$ si spezzi." Loc. cit., p. $866,1.5$.

$\dagger$ An explanation of this terminology, which was introduced by Noether, will be found in Severi, Lezioni di Geometria Algebrica, Chap. II.

$\ddagger$ Base points in the neighborhood of the first order are those which can be put in evidence by a single quadratic transformation having a fundamental point at $O_{1}$.

$\S$ Cf. footnote on p. 298 below.
} 
more, if there exist $\infty^{2}$ curves of the second degree passing through the points in question it is easily seen that they are not all reducible. Because if they were, their variable portion would be a general line of the plane and their fixed portion a line through $O_{0}, O_{1}$, and $O_{2}$, which is impossible since no line can meet a curve of the net in more than $n$ points. It is however false to assume that there always exist $\infty^{2}$ conics satisfying the given conditions.

\section{II}

4. Noether's theorem is equivalent to the theorem that a homaloidal net can always be carried into a net of straight lines by a series of quadratic transformations. Let $N$ be a homaloidal net of degree $n$ and let

$$
\alpha_{0} \geqq \alpha_{1} \geqq \cdots \geqq \alpha_{k} \geqq 1
$$

be the multiplicities of its base points

$$
O_{0}, O_{1}, \cdots O_{k}
$$

respectively, where (2) includes all the base points, ordinary and infinitely neighboring. We then bave the two well-known relations

and

$$
\sum_{i=0}^{k} \alpha_{i}^{2}=(n-1)(n+1)
$$

$$
\sum_{i=0}^{k} \alpha_{i}\left(\alpha_{i}-1\right)=(n-1)(n-2),
$$

the first expressing the condition that all but one of the intersections of two curves of the net occur at the base points, the second that the curves of the net are of genus zero. Multiplying (3) and (4) by $j-1$ and $j$ respectively, and subtracting the first from the second, we have

$$
\sum_{i=0}^{k} \alpha_{i}\left(\alpha_{i}-j\right)=(n-1)(n-3 j+1) ;
$$

and since, when we put $j=\alpha_{0}$, all the terms of the sum are zero or negative, we have

$$
0 \geqq(n-1)\left(n-3 \alpha_{0}+1\right),
$$

from which we conclude that

$$
\alpha_{0} \geqq \frac{n+1}{3}>\frac{n}{3}
$$

5. From now on, we shall put

$$
j=\frac{n-\alpha_{0}}{2} \geqq \frac{1}{2}, \quad \text { or } \quad n=\alpha_{0}+2 j,
$$


and shall denote by $h$ the number of base points other than $O_{0}$ of multiplicities greater than $j$. The multiplicities of these points are limited by the inequalities

$$
2 j \geqq \alpha_{1} \geqq \alpha_{2} \cdots \geqq \alpha_{h}>j
$$

because the line $O_{0} O_{1}$ can only meet a curve of the net $N$ in $2 j$ points aside from $O_{0}$. Moreover, their number is always greater than 1 except for a net of lines, as the following argument will show.

From the right-hand member of (5), we drop off the positive quantity $3 j-1$ and from the other, all the terms of the summation after the one in $\alpha_{h}$, that is, all the terms which are negative or zero. We then have

$$
\sum_{i=0}^{n} \alpha_{i}\left(\alpha_{i}-j\right)>n(n-3 j)=n\left(\alpha_{0}-j\right) ;
$$

or, on transposing the first term of the sum,

$$
\sum_{i=1}^{h} \alpha_{i}\left(\alpha_{i}-j\right)>\left(n-\alpha_{0}\right)\left(\alpha_{0}-j\right)=2 j\left(\alpha_{0}-j\right) .
$$

Then, on account of (9), we have $a$ fortiori

and therefore,

$$
2 j \sum_{i=1}^{h}\left(\alpha_{i}-j\right)>2 j\left(\alpha_{0}-j\right),
$$

$$
\sum_{i=1}^{h}\left(\alpha_{i}-j\right)>\alpha_{0}-j=n-3 j .
$$

Finally, since $\alpha_{1} \geqq \alpha_{0}$,

$$
h \geqq 2 .^{*}
$$

We also have from (10)

so that

$$
\sum_{i=1}^{h} \alpha_{i}>n+(h-3) j,
$$

$$
\sum_{i=1}^{h} \alpha_{i}>n \quad \text { when } \quad h \geqq 3,
$$

an inequality which we shall have occasion to use presently.

6 . It will be convenient to let the integer $2 j$ be a measure of the complexity of the net $N$. The simplest nets after the linear ones will then be those for which $2 j=1 . \dagger$ With this convention, it will be shown that the net $N$ can always be simplified (and therefore finally reduced to a net of lines) by a

* This is one way of showing that the sum of the multiplicities of $O_{0}, O_{1}$, and $O_{2}$ is greater than $\alpha_{0}+2 j=n$.

$\dagger$ These are the nets defining the so-called Jonquières transformations. They have a base point of order $\nu-1$ and $2 \nu-2$ simple base points. The proof from now on is a direct generalization of Segre's method for reducing Jonquières nets. 
series of quadratic transformations. In simplifying the net, we shall make use only of quadratic transformations of the involutoric type with three distinct base points $A, B$, and $C$, each of which is carried into the line through the other two. Moreover, we shall always choose the point $O_{0}$ as the base point $A$, so that the pencil of lines through $O_{0}$ will at each stage be transtormed into itself. Under these conditions, the complexity $2 j$ of each successive net will remain invariant just as long as the point $O_{0}$ remains one of the points of highest multiplicity, being measured by the number of variable points of intersection of a line through $O_{0}$ with a curve of the net. If, however, the point $O_{0}$ ever ceases to be the point of highest multiplicity, the net $N^{\prime}$ appearing at that stage will be simpler than $N$, for its complexity will be measured by $2 j^{\prime}$, the number of free points of intersection with $N^{\prime}$ of a line through a point of higher multiplicity than $O_{0}$. It is evident that $2 j^{\prime}$ is less than $2 j$.

7. Suppose to begin with that two points $O_{x}$ and $O_{y}$ of the set

$$
\left(O_{1}, O_{2}, \cdots O_{h}\right)=\left[O_{i}\right]_{1}^{h}
$$

of multiplicities greater than $j$ are distinct from one another and from $O_{0}$. They can then be dispersed by a quadratic transformation with $O_{0}, O_{x}$, and $O_{y}$ as base points. Furthermore, the two new base points which are ordinarily introduced at $O_{x}$ and $O_{y}$ are certainly each of multiplicities less than $j$, because the lines $O_{0} O_{x}$ and $O_{0} O_{y}$ meet the curves of $N$ in at most $n-\alpha_{0}-\alpha_{h}<j$ points. A single transformation will therefore reduce by two the number of base points of multiplicities greater than $j$. Moreover, if two of the remaining points of the set $\left[O_{i}\right]_{1}^{h}$ are distinct from one another and from $O_{0}$, we can disperse them in turn by a second transformation, and so on, until less than two remain. But the net $N^{\prime}$ appearing at this stage must now be simpler than $N$, for if $2 j^{\prime}$ were equal to $2 j$, we should be led into a contradiction with (11). In the particular case where $2 j$ is unity, the net $N^{\prime}$ must consist of straight lines.

8. There remains to be considered the case where at some stage, which we can suppose to be the first, no two points of the set $\left[O_{i}\right]^{h}$ are distinct from one another and from $O_{0}$. Our first quadratic transformation will then be made with one base point at $O_{0}$, and the two others $A$ and $B$ in general position. Instead of dispersing any of the points $\left[O_{i}\right]_{1}^{h}$, this transformation will introduce two new ones of multiplicities $2 j>j$, one at $A$ and one at $B$. On the other hand, it will detach from $O_{0}$ all its infinitely neighboring base points, transforming them into points on and infinitely neighboring to the line $A B$. If after the transformation there is a point $O^{\prime \prime}$ of $\left[O_{i}\right]^{h+2}$ infinitely neighboring to another $O^{\prime}$, we shall apply a second quadratic transformation with base points at $O_{0}, O^{\prime}$, and some third point $C$ in general position. This 
one will disperse $O^{\prime}$, putting $O^{\prime \prime}$ in evidence somewhere along $O C$, but at the same time it will introduce at $O^{\prime}$ a new base point of multiplicity $2 j$. It will therefore not alter the number of points in the set $\left[O_{i}\right]_{1}^{h+2}$ while it will increase by one the number of distinct ones. Since the line $O_{0} O^{\prime}$ is a fundamental line of the transformation, the last statement would not be true if there could be points of $\left[O_{i}\right]^{h+2}$ other than $O^{\prime}$ along $O_{0} O^{\prime}$, nor would it be possible to put $O^{\prime \prime}$ in evidence it it were infinitely neighboring to $O^{\prime}$ along $O_{0} O^{\prime}$; but neither of these cases can arise, since the sum of the multiplicities of any two points of $\left[O_{i}\right]^{h+2}$ exceeds $2 j$. By a finite number of steps like the last, we can therefore pass to a net with only $h^{\prime}=h+2$ points other than $O_{0}$ of multiplicities greater than $j$, all of which will be distinct. We then observe by (12), where $h^{\prime}$ now replaces $h$ in the tormula, that the points $\left[O_{i}\right]^{h^{\prime}}$ are not all collinear, so that the next quadratic transformation can be made to disperse a pair of them while leaving at least two others distinct from one another and from $O_{0}$. A second pair can therefore be dispersed at the next step, after which there will be two less points in the set than there were at the very start. Repeating the whole process a sufficient number of times, we can thus cut down the number of points of the set $\left[O_{i}\right]$ to less than two, when we shall be back to the case previously considered. It is therefore clear that any net may be simplified by successive stages and finally reduced to a net of straight lines.

Princeton University, November, 1915 